7 Charles Goodhart, Rosa Lastra Populism and Central Bank Independence. Open Economics Review. February 2018, Volume 29, Issue 1, pp 49-68.

8 Cruzio Giannini. The Age of Central Banks. Published by EH.Net. - 2011. - p. 132.

9 Nils Herger. Understanding Central Banks. Published by Springer. - 2019. - pp. 185-186.

10 Miao Han. Central bank Regulation and the Financial Crisis. Published by Palgrave Macmillan. 2016. - pp. 262-267.

11 Annual Report 2017. International organization of securities commissions. Интернет-ресурс. URL: https://www.iosco.org/annual_reports/2017. (Дата обращения: 24.09.2019)

\title{
SOME APPROACHES TO THE PHENOMENON OF BANKING SYSTEM REGULATION: RUSSIAN AND FOREIGN PRACTICE
}

\author{
(C) 2019 Anastasiya Dmirtievna Sterlikova \\ Postgraduate Student \\ Samara State University of Economics \\ E-mail: Sterlikova.A.D@gmail.com
}

Keywords: central bank, mega regulator, foreign practice, advantages, disadvantages.

This article includes some approaches of different authors to the phenomenon of mega regulation. It contained advantages and disadvantages of banking system regulation. It considered some risks of regulation in banking system. The conclusion about the impact of mega regulation on banking system is based on the analysis of various points of view.

УДК 33.338.36

Код РИНЦ 06.00.00

\section{МЕТОДИЧЕСКИЕ ПОДХОДЫ К АНАЛИЗУ ВОВЛЕЧЕННОСТИ ПРОМЫШЛЕННЫХ ПРЕДПРИЯТИЙ И ПРЕДПРИНИМАТЕЛЕЙ В МИРОВЫЕ ВОСПРОИЗВОДСТВЕННЫЕ ЦЕПОЧКИ}

\author{
() 2019 Стрельцов Алексей Викторович \\ доктор экономических наук, профессор \\ (с) 2019 Яковлев Геннадий Иванович \\ доктор экономических наук, профессор \\ Самарский государственный экономический университет \\ E-mail: dmms7@rambler.ru
}

Ключевые слова: промышленность, предпринимательство, индустриализация, воспроизводственные цепочки, стоимость, производительные силы.

В условиях явной недостаточности существующего методического аппарата и практического приложения традиционных экономических законов для удовлетворительного объяснения глобаль- 
ным переменам, протекающим в мировом хозяйстве, требуется разработать и обосновать новые принципы и направления организации воспроизводственной деятельности предприятий в рамках феномена "Четвертой промышленной революции". Происходит усиление глобального имущественного неравенства и трансформация мировых воспроизводственных цепочек создания стоимости, генерируемых промышленностью и предпринимателями, что обязывает учесть циклический характер производства новой потребительской стоимости в соответствии со стадиями ее жизненного цикла, и закономерностей механизма транснационального переноса новых технологий их производства в соответствии со стадиями индустриализации.

В современном мировом хозяйстве наблюдаются процессы замедления процессов глобализации, что особенно четко можно проследить по динамике международной торговли, выросшей на значительные 35\% с 1960 года по 2008 год, и всего лишь на 0,2\% за истекшие пять лет, отмечено в исследовании The New Globalization, 20171. При этом, если ряд экспертов выдвигают гипотезу о конце глобализации под влиянием беспрецедентного роста протекционизма, повышения стоимости труда в развивающихся странах, решорингу и политики новой индустриализации развитых стран, то работы В.Кондратьева говорят о ее новой модели на основе формирования новых пропорций сил ее акторов, дезинтеграции материальной составляющей цепочек формирования стоимости, стимулируемой технологиями индустрии 4.0, дифференциации путей роста развитых экономик и увеличения доли услуг в международной торговле2.

Внимание современных исследователей во всем мире сосредотачивается на таких проблемах, как структурные сдвиги в производственных трансграничных цепочках, создания добавленной стоимости и цифровизации производства, потребовавшие соответствующего уточнения методического и категориального аппарата. На уровне ООН даже была опубликовано в 2015 году "Руководство по измерению глобального производства" (Guide to Measuring Global Production, 2015) ${ }^{3}$, наряду с работами таких авторов, как Варнавский В. (2016) 4 , Hernández V., Pedersen T. (2017)5 анализировать существующие тенденции и масштабы участия промышленных предприятий и отдельных предпринимателей в глобальных воспроизводственных цепочках, чтобы оценить возможности и перспективы повышения роли отечественной экономики в международных интеграционных процессах. При этом данный анализ должен вскрыть как существующее положение дел, так и исследовать динамику данного процесса, наметить основные направления его совершенствования.

Научной новизной и практически значимой данное направление исследования способно обладать при условии содержательного теоретико-методического обоснования целенаправленной деятельности органов управления экономикой по формированию и развитию производственного и внешнеэкономического потенциала предприятий, пригодного для участия в мировых воспроизводственных цепочках. При этом должны обосновываться методические подходы оценки вклада в экономический рост как результатов развития производственной базы предприятий, так и условий функционирования регионального хозяйственного комплекса, примеров запуска крупных инвестиционных проектов, наращивания объемов несырьевого экспорта, предусматривающей как локализацию и импортозамещение промышленной продукции, так и кооперацию с фирмами ведущих стран при производстве конкурентоспособной наукоемкой продукции. 
В качестве теоретико-методологической основы исследования новых явлений в развитии мировых производительны сил предлагается использовать принципы комплексного и компаративного анализа, позволяющие охватить широкий ряд аспектов динамики и особенностей развития промышленного комплекса и предпринимательства различных стран, состояния национальной экономики и ее внешнеторгового комплекса; системный подход при определении мер, принимаемых при решении проблемы увеличения эффективности участия отечественных предприятий в мировых цепочках создания стоимости, сопровождаемое оценкой состояния их воспроизводственного аппарата. Исследование согласовывается с методологией М.Портера (1993)6 , в тех положениях, что конкурентное преимущество предприятий отдельных стран вырастает из всей системы видов их деятельности, причем успех определяется не столько наличием фракторов производства, но также степенью их продуктивного использования, особенно в обрабатывающих секторах, и соотнесенной величины добавленной стоимости.

Поэтому, представляется, что неотъемлемой частью анализа проблем, связанных с участием отечественных предприятий промышленности в глобальных воспроизводственных цепочках, должно являться изучение тенденций развития промышленности в целом, инвестиционных аспектов, воспроизводственных характеристик, понимаемых как исследование движения основного капитала, более подробная оценка особенностей развития "машиностроительных" видов экономической деятельности. Последнее представляется особо важным, поскольку именно машины и оборудование занимают большую долю импорта (55-60\%), представляют собой достаточно сложную инновационноемкую продукцию обрабатывающих производств, косвенно характеризуют уровень научнотехнического развития экономики. Следует также учитывать, что машины и оборудование составляют большую часть экспорта промышленно-развитых стран, и для обеспечения международного сотрудничества отечественных промышленных предприятий на равноправной основе необходимо соответствующее развитие машиностроения и отраслей, производящих продукцию с высокой добавленной стоимостью.

Следует учитывать также еще одно обстоятельство. В связи с неравномерным трендом развитием промышленности РФ в различные периоды времени С начала рыночных преобразований возникает вопрос - достаточно ли будет для достоверного анализа рассмотрение данных за несколько последних лет? Если нет, то какой период времени следует выбрать для получения реальной картины происходящего в мировых воспроизводственных цепочках? Представляется, что как раз последние годы (2018г., частично 2016, 2015 гг.) являются мало-показательными в плане раскрытия каких-либо тенденций. Это связано с тем, что на внешнеэкономические связи, да и в целом на экономическую деятельность в эти годы начали оказывать своё влияние разного рода санкционные ограничения, характер которых и последствия было трудно спрогнозировать и в то время, и сегодня, справедливо отмечают Клинова М.В., Сидорова Е.А. ${ }^{7}$. Анализ динамики показателей в эти годы также остается важным, но в первую очередь для того, чтобы показать, как менялись характеристики развития промышленности и её предприятий в условиях санкционных вызовов и взаимных ограничений. Для выявления каких-либо тенденций, очевидно, необходимо выбрать достаточно большой, репрезентативный период времени, как базовый интервал в 10-12 лет, в тоже время по ряду показателей можно использовать более короткие и даже "точечные" периоды времени. 
Вывод. В условиях глобализации ни одна страна в одиночку не сможет рассчитывать на ускоренные темпы роста и прогресса, и уход в изоляцию означает признание своей слабости и неконкурентоспособности. Адекватная методология анализа мировых воспроизводственных процессов является важной исходной базой в построение прогнозных оценок вовлеченности предприятий в существующие цепочки создания стоимости, позволяет выявить характеристики внешнеэкономической деятельности промышленных предприятий и предпринимательских структур, особенности динамики экспорта за достаточно длительный период исследования.

1 The new Globalization/ Going beyond the Retoric. Avialable at: https://www.bcg.com/publications/2017/ new- globalization-going-beyond-retoric (accessed 14.09.2019)

${ }^{2}$ Кондратьев В. Новый этап глобализации: особенности и перспективы/Мировая экономика и международные отношения. 2018. Том. 62. №6. С.5.

${ }^{3}$ Guide to Measuring Global Production. New York and Geneva. UNECE.2015.227 p. Available at: http://www.unece.org/statistics/publications/economic-statistics/national-accounts/2015/guide-tomeasuring-global-production-2015.html (accessed 10.09.18)

${ }^{4}$ Варнавский В. Международная торговля в категориях добавленной стоимости: вопросы методологии// Мировая экономика и междун. отн. 2018. Том. 62. №1. С.5-15.

${ }^{5}$ Hernández V., Pedersen T. Global value chain configuration: a review and research agenda// brq business research quarterly. Elsevier Science Publishing Company, Inc. ISSN: 2340-9436.Tом: 20 № 2 Год: 2017 C. DOI: 10.1016/j.brq.2016.11.001

${ }^{6}$ Портер, М. (1993). Международная конкуренция. Москва: Межд. отношения, с.680.

7 Клинова М.В., Сидорова Е.А. Экономические санкции Запада против России: развитие ситуации//Проблемы прогнозирования, 2019, №3. С.159.

\title{
METHODOLOGICAL APPROACHES TO THE ANALYSIS OF THE INVOLVEMENT OF INDUSTRIAL ENTERPRISES AND ENTREPRENEURS IN THE GLOBAL REPRODUCTION CHAIN
}

\author{
(c) 2019 Streltsov Alexey Victorovich \\ Doctor of Economics, Professor \\ (C) 2019 Yakovlev Gennady Ivanovich \\ Doctor of Economics, Professor \\ Samara State University of Economics \\ E-mail: dmms7@rambler.ru
}

\begin{abstract}
Keywords: industry, entrepreneurship, industrialization, reproduction chains, value, productive forces.

In the conditions of obvious insufficiency of the existing methodical apparatus and practical application of traditional economic laws for a satisfactory explanation of the global changes occurring in the world economy, it is required to develop and justify new principles and directions of the organization of reproductive activity of enterprises within the phenomenon of the "Fourth industrial revolution". There is an increase in global property inequality and the transformation of the world's reproductive value chains generated by industry and entrepreneurs, which obliges to take into account the cyclical nature of the production of new consumer value in accordance with the stages of its life cycle, and the laws of the
\end{abstract}


mechanism of transnational transfer of new technologies of their production in accordance with the stages of industrialization.

УДК 33.338.36

Код РИНЦ 06.00.00

\title{
ОСОБЕННОСТИ УЧАСТИЯ ПРОМЫШЛЕННЫХ ПРЕДПРИЯТИЙ В МИРОВЫХ ВОСПРОИЗВОДСТВЕННЫХ ЦЕПОЧКАХ В ФОРМАТЕ ОСОБЫХ ЭКОНОМИЧЕСКИХ ЗОН
}

\author{
(с) 2019 Стрельцов Алексей Викторович \\ доктор экономических наук, профессор \\ (c) 2019 Яковлев Геннадий Иванович \\ доктор экономических наук, профессор \\ Самарский государственный экономический университет \\ E-mail:dmms7@rambler.ru
}

\begin{abstract}
Ключевые слова: особые экономические зоны, преференции, льготный режим хозяйствования, интеграция.

Рассматриваются особенности деятельности и проблемы эффективной организации особых экономических зон (ОЭЗ), необходимые для ускорения вовлечения предприятий и национальных факторов производства в глобальные воспроизводственные цепочки. Показано, что значительные бюджетные средства, вложенные в их создание, приводят только к отдельным, точечным результатам, в противовес к сформировавшейся положительной международной практике. Необходимо четко определиться с реализацией целей функционирования отдельных зон, стратегии работы с инвесторами.
\end{abstract}

В специальной литературе и на практике все больше усиливается внимание к проблемам формирования и функционирования международных производственных цепочек, пересекающих границы государств и их интеграционных объединений. Важность исследований в данной области подтверждаются научными положениями, изложенными в работах таких ученых, как Варнавский В. (2016)1, Сапир Е.В. (2016)2, Резников С.Н. (2014)3, Кашбразиев P.B. (2015)4, Hernández V. и Pedersen T. (2017)5, Clarysse В. и др. (2014)6, Bremmer J. (2014)7, Strange R., и Magnani G. (2018)8, Laplume A. и др. (2016)9. Внимание современных исследователей сосредотачивается на таких проблемах, как структурные сдвиги в производственных трансграничных цепочках, создания добавленной стоимости и организация мультинационального производства, потребовавшие соответствующего уточнения методического и категориального аппарата, более полно отражающего феномен "Индустрии 4.0".

При этом следует учитывать, что системная статистическая инфрормация об участии предприятий промышленности в глобальных воспроизводственных цепочках является достаточно ограниченной в связи с отнесением данных по этому виду деятельности, 